\title{
TEST OF THE ELECTRON HOSE INSTABILITY IN THE E157 EXPERIMENT*
}

\author{
B. Blue, C. E. Clayton, E. Dodd, K. Marsh, W. B. Mori, S. Wang, and C. Joshi, UCLA, Los Angeles, CA 90095 \\ R. Assmann, CERN, Geneva, Switzerland \\ F-J. Decker, M. J. Hogan, R. H. Iverson, P. Raimondi, D. Walz, and R. H. Siemann, SLAC, Menlo Park, CA 94025 \\ S. Lee, P. Muggli, and T. Katsouleas, USC, Los Angeles, CA 90089
}

\begin{abstract}
The E157 experiment is designed to demonstrate highgradient Plasma Wake Field Acceleration over a significant length. It has been suggested that the electron hose instability of the drive beam will degrade the performance of this experiment because the hosing tailelectrons will not fully sample the highest acceleration field. In this paper a parasitic experiment designed to test the extent of the hosing instability is described. In particular, we discuss how the initial beam conditions are determined so that the extent to which any transverse perturbations grow due to hosing can be determined.
\end{abstract}

\section{DETERMINATION OF THE INITIAL CONDITIONS FOR ELECTRON HOSE INSTABILITY EXPERIMENT}

In this paper we discuss how we determine the transverse offsets of the various longitudinal beam slices before the beam enters the plasma source in the E157 experiment. Such a well characterized beam is then sent through the plasma that is roughly $1.4 \mathrm{~m}$ long with a maximum density of about $2 \times 10^{14} \mathrm{~cm}^{-3}$ to see if there is any growth of the transverse offsets of the beam slices due to hosing.

\section{MOTIVATION}

The electron hose instability [1] of the drive beam is of some concern to the plasma wake field accelerator concept because it can lead to the growth of transverse perturbations on the beam due to the nonlinear coupling of the beam electrons to the plasma electrons that are at the edge of the plasma channel through which the beam propagates. The instability is described by two coupled wave equations:

$$
\begin{aligned}
& \partial_{\mathrm{s}}^{2} \mathrm{x}_{\mathrm{b}}+\mathrm{k}_{\beta}^{2} \mathrm{x}_{\mathrm{b}}=\mathrm{k}_{\beta}^{2} \mathrm{x}_{\mathrm{c}} \\
& \partial_{\xi}^{2} \mathrm{x}_{\mathrm{c}}+\omega_{\mathrm{o}}^{2} \mathrm{x}_{\mathrm{c}}=\omega_{\mathrm{o}}^{2} \mathrm{x}_{\mathrm{b}}
\end{aligned}
$$

where $\xi=\mathrm{z} / \mathrm{c}-\mathrm{t}, \mathrm{s}=\mathrm{z}$, and $\omega_{\beta} / \mathrm{k}_{\beta}=\mathrm{c}$ with $\omega_{\beta}$ being the usual betatron frequency. Subscripts $c$ and $b$ refer to the ion channel and the beam, respectively. We can see that the beam centroid $x_{b}$ is driven by the channel centroid $x_{c}$ and vice versa. The frequency of oscillation along the

*Work supported by DOE No. DE-FG03-92ER40727 and NSF No. ECS 9617089 length of the beam is $\omega_{\mathrm{o}}=\omega_{\mathrm{p}} / \sqrt{2}$ where $\omega_{\mathrm{p}}$ is the plasma frequency.

The growth of the transverse displacement $\mathrm{x}_{\mathrm{b}}$ of $\mathrm{a}$ longitudinal slice of the beam due to the electron hose instability has been calculated for a beam with a linear tilt with the asymptotic result: [2]

$$
\begin{aligned}
& x_{b}=0.341 \frac{x_{b_{0}}}{A^{3 / 2}} e^{A} \cos \left\{k_{\beta} s-\frac{A}{\sqrt{3}}+\frac{\pi}{12}\right\} \\
& \text { where } A=\frac{3^{3 / 2}}{4}\left[\left(k_{\beta} s\right)\left(\omega_{0} c\right)^{2}\right]^{1 / 3}
\end{aligned}
$$

Thus it is critical that the initial beam tilt $\mathrm{x}_{\mathrm{b}_{\mathrm{o}}}$ be extremely well known in any hosing experiment in order to measure the growth of this initial displacement.

\section{METHOD}

The experimental set-up of the E157 experiment has been described in detail elsewhere. [3] In this paper we describe only those aspects of the experiment that are relevant to determining the transverse offsets along the longitudinal profile of the beam at the entrance of the plasma source.

In general, the beam entering the final-focusing optics (a few meters before the plasma entrance) can have a headto-tail tilt along its (rms) length $\sigma_{z}$. For a tilt in the $\mathrm{x}$ plane, the optics will impart a momentum $\mathrm{P}_{\mathrm{x}}$ to a slice $\sigma_{\mathrm{z}}$ behind the centroid of the beam. If the longitudinal momentum of the beam is $\mathrm{P}_{z}$ and the effective focal length of the optics is $\mathrm{f}_{\text {eff }}$, then this tilt will be magnified by a factor $\left(\mathrm{P}_{\mathrm{x}} / \mathrm{P}_{\mathrm{z}}\right)\left(\mathrm{z} / \mathrm{f}_{\text {eff }}\right)$ at a distance $\mathrm{z}$ away from the focus of the beam (which is near the entrance to the plasma chamber). We assume that this transverse kick from the optics dominates over the range of transverse momenta prior to the optics, which is reasonable given the hundreds of meters between the linac (where the offsets originate) to the experiment.

The time-integrated electron beam profiles entering and exiting the plasma can be measured using opticaltransition-radiation (OTR) foils placed $1.2 \mathrm{~m}$ before (upstream or US-OTR) and $1.1 \mathrm{~m}$ after (downstream or DS-OTR) the $1.4 \mathrm{~m}$ plasma source which are viewed by 
two CCD cameras. Upon exiting the plasma the electron beam is dispersed in the y or vertical direction using a dipole magnet. The beam subsequently passes through a $1 \mathrm{~mm}$ thick aerogel Cherenkov radiator. The Cherenkov

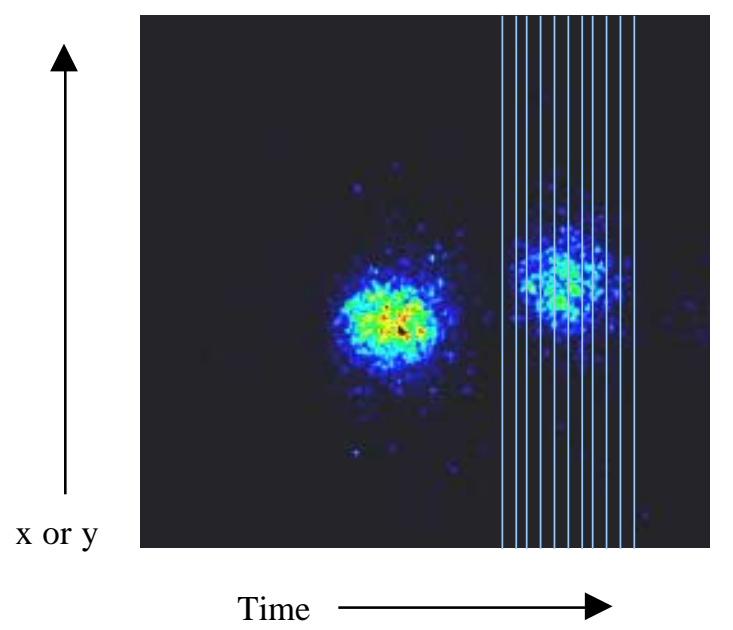

Figure 1: Typical streak camera data. The left streak image is $y(t)$ and the right is $x(t)$. The lines on the image illustrate how the data-analysis software slices up the time-resolved image into 1-psec bins.

radiation profile is also imaged on a CCD camera to obtain the time integrated beam spot size. The spot size $\sigma_{\mathrm{x}}$ in the $\mathrm{x}$ or the horizontal plane at the Cherenkov plane does not have any contribution due to energy spread on the beam (as does the y size $\sigma_{y}$ ). A fraction of the Cherenkov light is time resolved using a streak camera to give the time history of the transverse features of the beam during a single pulse.

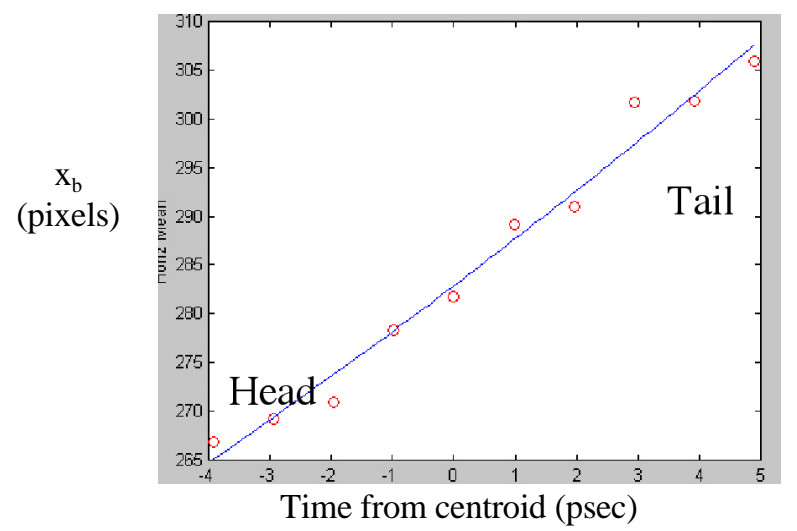

Figure 2: Analysis of the $\mathrm{x}(\mathrm{t})$ streak from Fig. 1 shows a typical tilted beam. The points are the transverse $\mathrm{x}$ displacement $x_{b}$ of ten 1-psec slices around the longitudinal centroid $(0 \mathrm{psec})$ of the bunch.

The initial tilt on the beam in the horizontal plane is estimated by analyzing the streak data from the horizontal plane (x vs. t). The first step is to slice up the streak image in psec bins as shown in Fig. 1 where the horizontal streak image has been selected for analysis. Next, each slice is analyzed to find its centroid position. This is the transverse displacement $x_{b}$ of each slice at this observation plane. Finally, a tilt implies that the $\mathrm{x}_{\mathrm{b}}$ of each slice will change in time.

The results of this analysis for the single event shown in Fig. 1 are plotted in Fig. 2 with the horizontal-axis being time in psec and the vertical-axis being the transverse displacement of each psec slice position in pixels on the CCD camera, with 1 pixel $=16.1 \mu \mathrm{m}$. In Fig 2, 0 psec is the longitudinal centroid of the beam. For this event, the beam head-to-tail tilt is approximately linear.

The results for a run of 25 consecutive shots are given in Figure 3. Every data point is plotted for each psec bin. The solid line drawn through the points represent the average of all 25 shots for each time step. The two dashed lines represent the average \pm standard deviation for each time step. The average tilt of the beam is not linear but apparently more like a "banana".

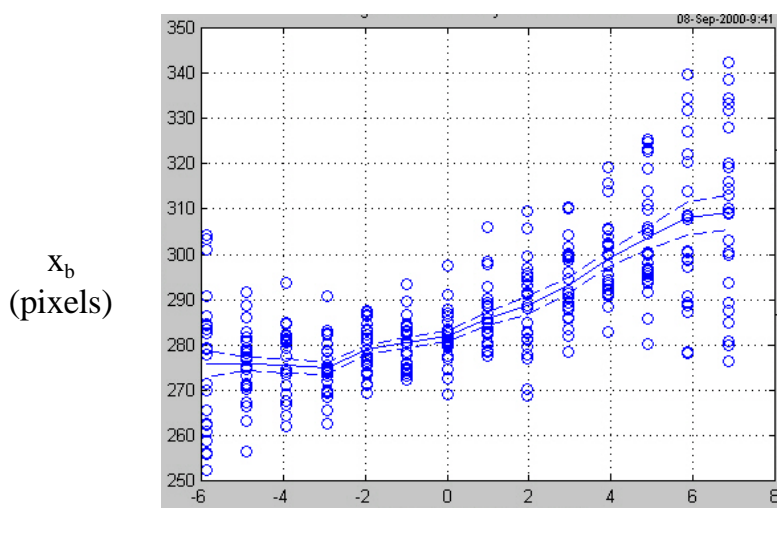

Time from centroid (psec)

Figure 3: Same as Fig. 2 except that 25 consecutive shots are plotted. The solid (dashed) line(s) is the average (standard deviation) of $\mathrm{x}_{\mathrm{b}}$ for the 25 shots.

From the streak data shown in Fig. 1, the bunch length $\sigma_{\mathrm{z}}$ is measured to be about $2 \mathrm{ps}$. The average beam offset $\mathrm{R}_{1}$ at a location $\sigma_{\mathrm{z}}$ behind the beam centroid at the Cherenkov plane is, from Fig. 3, about $100 \mu \mathrm{m}$. The average beam spot size $\sigma_{1}$ in the x-direction measured from the time-integrated Cherenkov images for the 25 shots was $650 \mu \mathrm{m}$. This gives a beam tilt normalized to the spot size of $\sigma_{1} / R_{1}=0.15$.

The measured tilt corresponds to the beam parameters at the aerogel that is 12 meters downstream from the plasma chamber. What we are interested in is the beam tilt at the entrance of the plasma. Ideally we would move the aerogel and the streak camera to the plasma entrance. This. however, is not possible because of experimental constraints, so we approximate the beam tilt at the plasma entrance. We do this by fitting the beam envelope equation: 


$$
\begin{aligned}
& \frac{\partial^{2} \sigma(z)}{\partial z^{2}}+\left[K^{2}-\frac{\varepsilon_{N}^{2}}{\gamma^{2} \sigma^{4}(z)}\right] \sigma(z)=0 \\
& K=\left\{\begin{array}{cc}
0 & \text { no plasma } \\
\frac{\omega_{p}}{c \sqrt{2 \gamma}} & \text { in a plasma }
\end{array}\right.
\end{aligned}
$$

to the three known spot sizes (upstream OTR, downstream ORT, and time-integrated Cherenkov) in order to find the spot size at the plasma entrance. Here $\varepsilon_{\mathrm{N}}$ is the normalized emittance. Figure 4 shows the results of fitting Eq. (1) to the 3 measured spot sizes. We find that

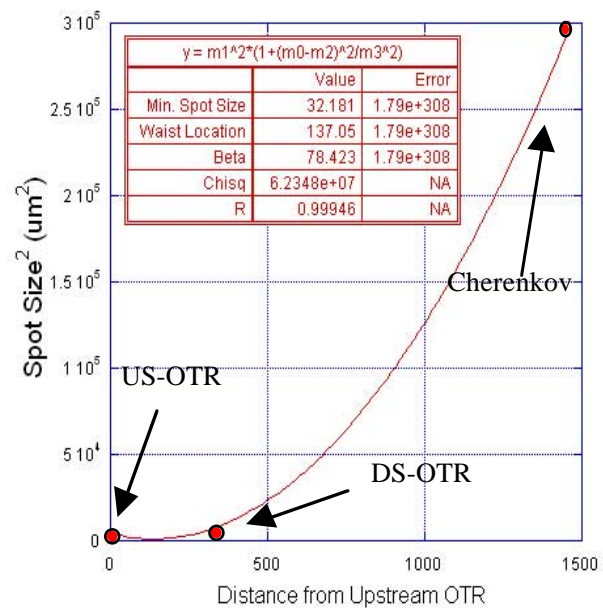

Figure 4: A beam-envelope fit to 3 measured spot sizes giving the location and size of the beam waist.

the beam waist is located approximately $20 \mathrm{~cm}$ from the plasma entrance with a minimum spot size of about 32 $\mu \mathrm{m}$. After determining the spot size at the plasma entrance, we determine the offset $R_{1}$ at the plasma entrance by scaling the offset at the aerogel $R_{1}$ by the ratio of the two beam spots sizes as illustrated in Fig. 5.

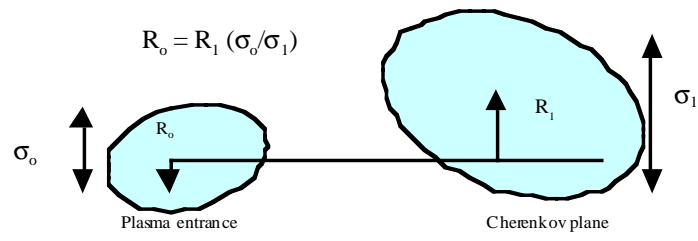

Figure 5: Scaling of beam offset or tilt from the Cherenkov location to the entrance of the plasma.
Applying this technique to all 25 shots of Fig. 3, the values for the initial offset of the beam slice $x_{b_{o}}$ at one $\sigma_{z}$ behind the centroid at the plasma entrance are as shown in Fig. 6.

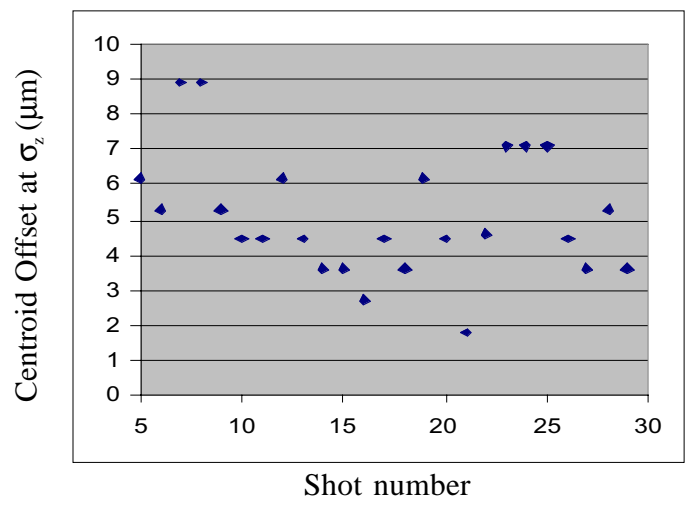

Figure 6: Tail offset (at $\sigma_{\mathrm{z}}$ after centroid) at the plasma entrance for 25 shots.

One can see that the mean "tail" ( $\sigma_{\mathrm{z}}$ after centroid) offset is typically $\sim 5 \mu \mathrm{m}$ which is much smaller than the radius $\sigma_{\mathrm{r}}$ of the beam. Therefore the tilt is not usually identified on the time integrated OTR diagnostics for a well-tuned beam in the absence of the plasma.

We have subsequently sent such beams through 1.4 meter long lithium plasmas of densities in the range $10^{12}-2 \times 10^{14} \mathrm{~cm}^{-3}$ to see if the transverse perturbations are affected by the hosing instability. These results will be published elsewhere.[4]

\section{ACKNOWLEDGMENTS}

We thank P. Catravas, W. Leemans, D. Whittum and J. Rokni for their contributions to this work.

\section{REFERENCES}

[1] D. H. Whittum, Physical Review Letters 67, 991 (1991).

[2] A. A. Garaci and D. M. Whittum, Physics of Plasmas 7, 3431 (2000).

[3] M. J. Hogan et al., Physics of Plasmas 7, 2241 (2000).

[4] B. Blue et al., to be submitted for publication. 